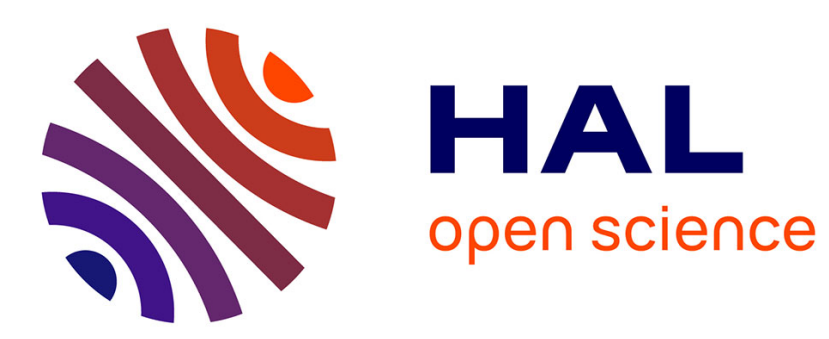

\title{
Adaptive Whole-Body Manipulation in Human-to-Humanoid Multi-Contact Motion Retargeting
}

Kazuya Otani, Karim Bouyarmane

\section{To cite this version:}

Kazuya Otani, Karim Bouyarmane. Adaptive Whole-Body Manipulation in Human-to-Humanoid Multi-Contact Motion Retargeting. HUMANOIDS 2017 - IEEE-RAS International Conference on Humanoid Robots, Nov 2017, Birmingham, United Kingdom. pp.1-8. hal-01569390v2

\section{HAL Id: hal-01569390 \\ https://hal.science/hal-01569390v2}

Submitted on 5 Dec 2017

HAL is a multi-disciplinary open access archive for the deposit and dissemination of scientific research documents, whether they are published or not. The documents may come from teaching and research institutions in France or abroad, or from public or private research centers.
L'archive ouverte pluridisciplinaire HAL, est destinée au dépôt et à la diffusion de documents scientifiques de niveau recherche, publiés ou non, émanant des établissements d'enseignement et de recherche français ou étrangers, des laboratoires publics ou privés. 


\section{Adaptive Whole-Body Manipulation in Human-to-Humanoid Multi-Contact Motion Retargeting}

Kazuya Otani

\begin{abstract}
We propose a controller for loco-manipulation motion retargeting from a human to a humanoid robot. Using this controller, the robot can track complex motions and automatically adapt to elements in the environment that have different physical properties from those that were used to provide the human's reference motion. The multi-contact loco-manipulation problem is formulated as a multi-robot quadratic program (MRQP), which optimizes over the combined dynamics of the robot and any manipulated element in the environment. Our approach maintains a dynamic partition of the robot's tracking links into fixed support contact links, manipulation contact links, and contact-free tracking links. The three sets are repartitioned and re-instantiated as objectives or constraints in the MRQP when contact events occur in the human motion. We present various experiments (bag retrieval, door opening, box lifting) using human motion data from an Xsens inertial motion capture system. We show in dynamics simulation that the robot is able to perform difficult single-stance motions as well as multi-contact-stance motions (including hand supports), while adapting to environment elements of varying inertial properties.
\end{abstract}

\section{INTRODUCTION AND RELATED WORK}

Recent developments in human motion tracking technologies allow high-fidelity, high-frequency tracking of a human's motion. The data from these systems can be used to generate human-like motions on humanoid robots.

Human-to-humanoid motion retargeting can be applied to teleoperation in disaster scenarios, where a robot may be better equipped to enter hazardous environments, as well as learning from demonstration, in which a robot learns a generalizable representation of a task from human demonstration. In particular, we believe that motion retargeting is a powerful tool for bootstrapping robot motion planning in complex multi-contact scenarios. Examples of tasks in this category are the vehicle egress task in the DARPA Robotics Challenge, and walking through cluttered manufacturing environments.

However, direct mapping of motion from humans to humanoid robots is not possible, due to differences in kinematics (e.g. joint limits, limb lengths) and dynamics (e.g. mass distribution). A motion retargeting method must generate a robot motion that is feasible and stable, while also following the salient parts of the human demonstration as closely as possible.

e-mail: kazuya.otani@inria.fr, karim.bouyarmane@loria.fr

The authors have the following affiliations:

- Inria, Villers-lès-Nancy, F-54600, France

- CNRS, Loria, UMR 7503, Vandœvre-lès-Nancy, F-54500, France

- Université de Lorraine, Loria, UMR 7503, Vandœuvre-lès-Nancy, F-54500, France

This work received funding from the European Commission (GA no. 731540, H2020 project “An.Dy").

\author{
Karim Bouyarmane
}

Pollard et al [1] showed one of the first approaches to motion retargeting of human movements for humanoids, in which they edited the human reference motion to keep the robot within its joint angle and velocity limits. They only imitated upper-body movements of a humanoid, and hence did not consider balance.

Subsequent works proposed methods for ensuring the robot's balance. Many of them use simplified models of the robot to generate center-of-mass $(\mathrm{CoM})$ trajectories and/or keep the robot stable around a desired CoM position. Dariush et al [2] formulated upper body imitation as task space control with a separate ZMP-based balance controller. Yamane and Hodgins [3] employed a "dynamics filter" to modify the CoM trajectory to keep the robot balanced. Kim et al [4] extracted footsteps from motion capture data and generated $\mathrm{CoM}$ trajectories with a linear inverted pendulum model. $\mathrm{Hu}$ et al [5] combine ZMP control with an objective to stay close to human joint trajectories. Bin Hammam et al [6] use the resolved acceleration method for tracking whole-body motions in double stance. Note that these methods were applied only to walking or standing; because of this, they often had built-in assumptions that only the feet would make contact with the environment, and that the robot is on flat horizontal ground.

This work is concerned with retargeting whole-body manipulation motions that are integrated with locomotion. This presents additional challenges, such as changes in support contacts, and more notably, changes in dynamics due to the manipulated objects.

To deal with multiple (possibly non-coplanar) contacts on arbitrary links, our work builds on that of Di Fava et al [7], which uses a QP controller to track human motion with Cartesian space "tasks" and to reason explicitly about contact friction cones [8]. Early QP control work in computer graphics demonstrated multi-objective control for generating natural movements in animated characters [9][10]. In recent years, QP-based humanoid controllers have become ubiquitous due to their robustness, real-time speeds, and intuitive formulation. In the recent DARPA Robotics Challenge, a majority of the teams relied on a QP for their low-level fullbody controller [11][12][13].

In existing motion retargeting methods, the dynamics of manipulated objects are assumed to be external perturbations [14] or negligible [15] (note that in [14] the character is rooted to the ground and hence the manipulated objects do not affect its balance). Vaillant et al [16] introduced the idea of incorporating external objects and other characters into a single QP formulation, which allows explicit opti- 
mization over physically and behaviorally coupled systems. Bouyarmane et al [17] implemented this on multi-robot motions. Other works have shown approaches to modeling the spatial relationships between multiple agents: Ho et al [18] introduced the concept of an "interaction mesh" for retargeting multi-character motions to characters of different morphologies while preserving spatial relationships; Nakamura and Komura [19] extended this work to include balance and joint constraints for humanoid robots.

Our approach differs from existing work in human-tohumanoid motion retargeting where manipulated objects, if any, are light relative to the robot's own weight. We use a multi-robot quadratic program (MRQP) framework, which explicitly models the combined dynamics of the robot and manipulated element in the environment [16]. This allows the robot to manipulate heavy objects that significantly affect its dynamics. It can also generalize human demonstrations performed on an object of specific properties to situations with objects of different physical properties (e.g. mass, friction, spring constant). Other desired behaviors, such as collision avoidance between bulky or cumbersome manipulated objects and the robot/environment, can be enforced cleanly within this formulation. Using this framework, we extend the range of activities possible in human-to-humanoid motion retargeting.

To demonstrate the capabilities of our method, we show a simulated robot imitating human demonstrations in several whole-body loco-manipulation tasks. We show that the robot can adapt its movement to account for objects of varying properties.

The rest of this paper is organized as follows. In Section II we summarize multi-contact motion retargeting with a QP controller, as introduced in [7]. In Section III we introduce additionally the new multi-robot QP formulation for multicontact motion retargeting. It is experimentally applied in Section IV, where various whole-body manipulation tasks are retargeted from a human to humanoid robots in full-body dynamics simulation. Finally, Sections V and VI conclude the paper by discussing the limitations of our method and indicating directions for future work.

\section{BACKGROUND : MULTI-CONTACT MOTION CAPTURE TRACKING QP}

In [7], human-to-humanoid multi-contact motion retargeting with contact changes is addressed in a two-stage approach. In the first stage, the sequence of contact change events

$$
L=\left(\eta_{1}, \ldots, \eta_{\mu}\right),
$$

is extracted from offline processing of the recorded motion. A contact change event is encoded into the tuple $\eta_{j}=\left(l_{j}, s_{j}, t_{j}, \sigma_{j}\right)$ with $l_{j}$ the robot link index, $s_{j}$ the environment surface index, $t_{j}$ the timing of the contact change event, and $\sigma_{j} \in\{0,1\}$ a binary variable encoding the event type ( 0 for a contact breaking event, 1 for a contact making event).

In the second stage of the approach, the recorded motion is tracked online by a QP controller, by decomposing the links

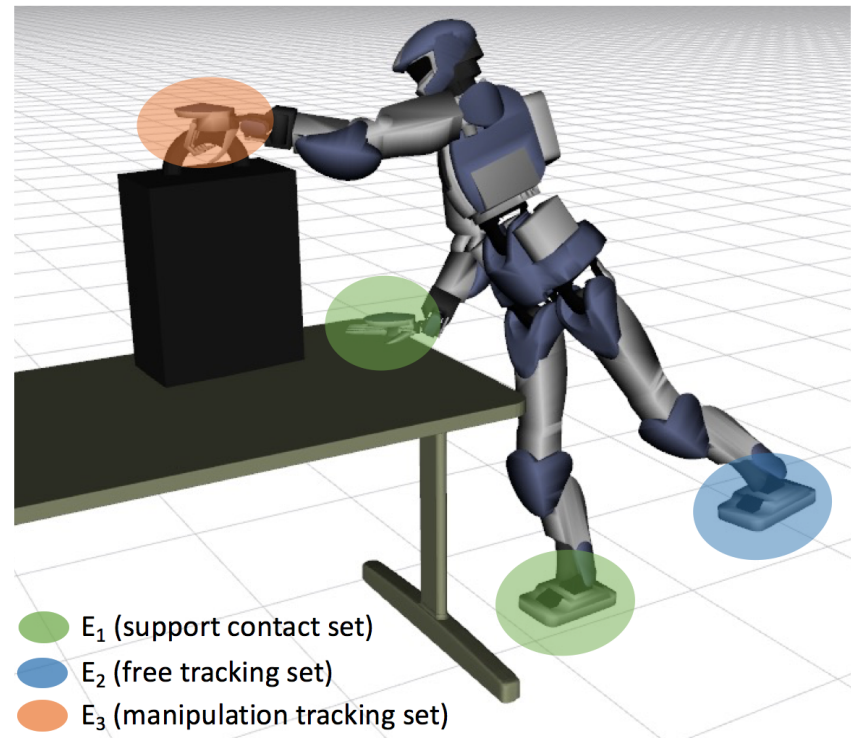

Fig. 1. Illustration of the contacts/tracking sets partition at a given phase of the bag retrieval motion. $E_{1}$ is the fixed contact supports set, which includes the right foot and the right hand. $E_{2}$ is the free tracking set, with only the left foot in this situation. $E_{3}$ is the manipulation tracking set, which includes the left hand holding the handle of the bag. At each contact event (addition or removal), the three sets $\left(E_{1}, E_{2}, E_{3}\right)$ are updated.

of the human/robot in two sets. $E_{1}$ is the contacts set, the set of links of the human/robot that are in contact with the environment. $E_{2}$ is the active tracking set, the set of links at the extremities the human/humanoid robot's limbs that are freely moving and could potentially make contact with external objects during the motion (we might refer to these as end-effectors). $E_{2}^{0}$ is the potential tracking set, a constant set that contains all the links that are tracked whenever they are not in contact, $E_{2} \subset E_{2}^{0}$ (note that links not belonging to $E_{2}^{0}$ can be in $E_{1}$, e.g. when sitting on the buttocks). The sets $E_{1}$ and $E_{2}$ are separate, i.e. $E_{1} \cap E_{2}=\emptyset$. For a given contacts/tracking sets distribution $\left(E_{1}, E_{2}\right)$, the QP that is instantiated for the robot to track the human motion is the following (we highlight the dependency on $\left(\boldsymbol{E}_{\mathbf{1}}, \boldsymbol{E}_{\mathbf{2}}\right)$ ):

$$
\begin{array}{r}
\min _{\ddot{q}, \tau, f_{\boldsymbol{E}_{1}}} \sum_{k \in \boldsymbol{E}_{\mathbf{2}}} w_{k}\left\|\ddot{x}_{k}-\ddot{x}_{k}^{d}\left(x_{k}^{\mathrm{hum}}\right)\right\|^{2} \\
+w_{c}\left\|\ddot{c}-\ddot{c}^{d}\left(h\left(\boldsymbol{E}_{\mathbf{1}}\right)\right)\right\|^{2} \\
+w_{0}\left\|\ddot{q}-\ddot{q}^{d}\left(q_{0}\right)\right\|^{2}, \\
\text { subject to } \quad M \ddot{q}+N=J_{\boldsymbol{E}_{1}}^{T} f_{\boldsymbol{E}_{1}}+S^{T} \tau, \\
J_{\boldsymbol{E}_{1}} \ddot{q}+\dot{J}_{\boldsymbol{E}_{1}} \dot{q}=0, \\
f_{\boldsymbol{E}_{1}} \in \mathcal{C}_{\boldsymbol{E}_{1}},
\end{array}
$$

torque limits, joint limits, collision avoidance,

where $q$ is the configuration of the robot, $M$ is the mass matrix of the robot, $N$ the nonlinear effects and gravity term, $\tau$ the actuation torques at the joints of the robot, $f_{E_{1}}$ the set of point contact forces applied at the contact links in $E_{1}$, $J_{E_{1}}$ the contact point Jacobians of the links in $E_{1}, S$ the allDoFs-to-actuated-DoFs selection matrix, $\mathcal{C}_{E_{1}}$ the Cartesian product of the linearized friction cones at the contact points 
in $E_{1}$.

The constraints for torque limits, joint angle/velocity limits, and collision avoidance (6) are described in [7], and are excluded here to emphasize the other equations which are dependent on the tracking/contact sets partition $\left(E_{1}, E_{2}\right)$.

In the cost function (2), the symbol $g \in\left\{{ }^{\prime} x_{k}\right.$ ', ' $c$ ', ' $q$ ' $\}$ denotes objectives, or "tasks": an end-effector pose (position, orientation) task, a center-of-mass (CoM) task, and a fullconfiguration task, respectively, with corresponding weights $w_{g}$ and stiffness $\kappa_{g}$. A task is specified by its desired acceleration $\ddot{g}^{d}\left(g^{\text {ref }}\right)$ that tracks a reference value $g^{\text {ref }}$ as:

$$
\ddot{g}^{d}\left(g^{\mathrm{ref}}\right)=\ddot{g}^{\mathrm{ref}}-\kappa_{g}\left(\dot{g}-\dot{g}^{\mathrm{ref}}\right)-2 \sqrt{\kappa_{g}}\left(g-g^{\mathrm{ref}}\right) .
$$

The reference value $x_{k}^{\text {ref }}$ for $k \in E_{2}$ is set at the corresponding link marker pose from the human motion capture data $x_{k}^{\text {hum }}$ :

$$
\forall k \in E_{2} \quad x_{k}^{\mathrm{ref}}=x_{k}^{\mathrm{hum}}
$$

The reference value for the $2 \mathrm{D}$ horizontal component of the CoM is set at $h\left(E_{1}\right)$, which denotes the center of the projected horizontal support polygon for the set of contacts in $E_{1}$ (i.e. the center of the convex hull of the ground projections of the contact points in $E_{1}$ ):

$$
c_{x, y}^{\mathrm{ref}}=h\left(E_{1}\right) \text {. }
$$

The vertical component $c_{z}^{\text {ref }}$ is set to track the human motion. Because maintaining balance is crucial to safe operation of the robot, the CoM task is given a high weight relative to the other tasks.

The reference of the full configuration task is set to a rest posture $q^{\text {ref }}=q_{0}$ with a low weight. This task ensures that the optimization is well-conditioned. In practice, it also helps generate more natural-looking movements.

The multi-contact motion retargeting algorithm uses the QP controller to track the human's motion, while reformulating the objectives and constraints of the QP every time a contact change event (i.e. a re-partitioning of $\left(E_{1}, E_{2}\right)$ ) is reached. For contact additions, a contact approach and stabilization phase is inserted in a fixed time window in order to ensure stable contacts.

\section{ADAPTIVE MANIPULATION MULTI-CONTACT MOTION RETARGETING}

In this work, we incorporate the dynamics of manipulated objects into the motion capture QP framework reviewed in Section II. In doing so, we make the following assumptions. First, we assume that any manipulated object's inertial and friction parameters are known to the robot. Second, it is assumed that the robot can identify relevant contact surfaces on the objects. These assumptions are reasonable if: the robot is operating in a known environment, a human operator can provide this information, or the robot has a perception system that can estimate these parameters. Given these assumptions, we can model the whole-body manipulation task in a multirobot QP (MRQP).

\section{A. Motion retargeting with manipulation as multi-robot $Q P$}

The main idea is to retarget environment manipulation motions performed by the human, to the humanoid robot manipulating its own environment. The environment of the robot may have different inertial properties from the environment being manipulated in the human motion capture data. The simplest example of such a situation is the human lifting a box of a certain mass in their demonstration, and the robot performing the same motion on boxes of different masses (demonstrated in Section IV). To achieve this, we integrate the MRQP into the multi-contact motion retargeting framework.

Suppose that the robot is manipulating an environment object, which may be a free-floating object (e.g. box), a fixed-base articulated mechanism (e.g. door, drawer), with passive joints, spring-loaded joints (knob of a door), or actuated joints (a second robot). As an abuse of language, we call all these objects "robots" (hence the "multi-robot" terminology). The general dynamical equation of motion of the environment object takes the form

$$
M_{o} \ddot{q}_{o}+N_{o}=J_{o}^{T} f_{o}+S_{o}^{T} \tau_{o},
$$

where $\tau_{o}$ are actuation torques. $q_{o}$ is the configuration of the environment object (e.g. the 6D position/orientation of a box, or the configuration of an articulated object plus its $6 \mathrm{D}$ base position/orientation if the base is not fixed). $f_{o}$ are all the external forces applied on the object. These include forces applied by the external fixed environment $\left(f_{e, o}\right)$ and the forces applied by the robot $\left(f_{r, o}\right): f_{o}=\left(f_{e, o}, f_{r, o}\right)$. Decomposing $f_{o}$, Equation (10) becomes

$$
M_{o} \ddot{q}_{o}+N_{o}=J_{e, o}^{T} f_{e, o}+J_{r, o}^{T} f_{r, o}+S_{o}^{T} \tau_{o} .
$$

Extending the framework described in Section II, we redefine $E_{1}$ as the support contacts set, the set of links of the robot that are in contact with the fixed environment. $E_{2}$ is now defined as the free tracking set, the set of limb extremities ("end-effectors") that are freely moving without any contact. We introduce a new set $E_{3}$ as the manipulation tracking set, the set of links of the robot that are in contact with the manipulated environment object. We denote $f_{E_{1}}$ as the forces applied by the fixed environment on the robot, and $f_{E_{3}}$ as the set of forces applied by the manipulated object on the robot. The robot's equation of motion (3) becomes:

$$
M \ddot{q}+N=J_{E_{1}}^{T} f_{E_{1}}+J_{E_{3}}^{T} f_{E_{3}}+S^{T} \tau .
$$

According to Newton's third law, the force applied by the robot on the object must be equal and opposite to the force applied by the object on the robot. Thus we have

$$
f_{E_{3}}=-f_{r, o} .
$$

The minimal set of forces of the problem are thus:

$$
f_{\text {multi-robot }}=\left(f_{e}, f_{E_{3}}\right) \text {, }
$$

where $f_{e}=\left(f_{E_{1}}, f_{e, o}\right)$ denotes the external forces applied by the fixed environment on the robot and the object, and $f_{E_{3}}$ the internal (to the combined system) forces exchanged 
between the robot and the object. We ensure that surfaces in contact move together with:

$$
J_{E_{3}} \ddot{q}+\dot{J}_{E_{3}} \dot{q}=J_{r, o} \ddot{q}_{o}+\dot{J}_{r, o} \dot{q}_{o}
$$

which complements both (4) and the following equation:

$$
J_{e, o} \ddot{q}_{o}+\dot{J}_{e, o} \dot{q}_{o}=0
$$

Let us now rewrite the motion capture tracking QP (2) as a MRQP for manipulating the environment object. The MRQP now tracks the links that are in $E_{2}$ (free tracking set) and in $E_{3}$ (manipulation tracking set). We define the multi-robot quantities by stacking the dynamics equations, as in [16]:

$$
\begin{aligned}
q_{\text {multi-robot }} & =\left(q, q_{o}\right), \\
M_{\text {multi-robot }} & =\operatorname{blockdiag}\left(M, M_{o}\right), \\
N_{\text {multi-robot }} & =\left(N, N_{o}\right), \\
S_{\text {multi-robot }} & =\operatorname{blockdiag}\left(S, S_{o}\right), \\
\tau_{\text {multi-robot }} & =\left(\tau, \tau_{o}\right) \\
J_{e} & =\operatorname{blockdiag}\left(J_{E_{1}}, J_{e, o}\right),
\end{aligned}
$$

The new motion capture tracking MRQP becomes (with subscript 'multi-robot' shortened to 'mr', and with dependency on set partition $\left.\left(\boldsymbol{E}_{\mathbf{1}}, \boldsymbol{E}_{\mathbf{2}}, \boldsymbol{E}_{\mathbf{3}}\right)\right)$ :

$$
\begin{aligned}
& \min _{\ddot{q}_{\mathrm{mr}}, \tau_{\mathrm{mr}}, f_{\mathrm{mr}}} \sum_{k \in \boldsymbol{E}_{\mathbf{2}} \cup \boldsymbol{E}_{\mathbf{3}}} w_{k}\left\|\ddot{x}_{k}-\ddot{x}_{k}^{d}\left(x_{k}^{\text {hum }}\right)\right\|^{2} \\
& +w_{c}\left\|\ddot{c}_{\mathrm{mr}}-\ddot{c}_{\mathrm{mr}}^{d}\left(h\left(\boldsymbol{E}_{\mathbf{1}}\right)\right)\right\|^{2} \\
& +w_{0}\left\|\ddot{q}-\ddot{q}^{d}\left(q_{0}\right)\right\|^{2}, \\
& M_{\mathrm{mr}} \ddot{q}_{\mathrm{mr}}+N_{\mathrm{mr}}=J_{e}^{T} f_{e}+ \\
& {\left[J_{\boldsymbol{E}_{3}}-J_{r, o}\right]^{T} f_{\boldsymbol{E}_{\mathbf{3}}}+S_{\mathrm{mr}}^{T} \tau_{\mathrm{mr}},} \\
& J_{\boldsymbol{E}_{1}} \ddot{q}+\dot{J}_{\boldsymbol{E}_{1}} \dot{q}=0, \\
& J_{e, o} \ddot{q}_{o}+\dot{J}_{e, o} \dot{q}_{o}=0, \\
& J_{\boldsymbol{E}_{\mathbf{3}}} \ddot{q}+\dot{J}_{\boldsymbol{E}_{\mathbf{3}}} \dot{q}=J_{r, o} \ddot{q}_{o}+\dot{J}_{r, o} \dot{q}_{o}, \\
& \left(f_{\boldsymbol{E}_{1}}, f_{\boldsymbol{E}_{\mathbf{3}}}, f_{e, o}\right) \in \mathcal{C}_{\boldsymbol{E}_{\mathbf{1}}} \times \mathcal{C}_{\boldsymbol{E}_{\mathbf{3}}} \times \mathcal{C}_{e, o},
\end{aligned}
$$

torque limits, joint limits, collision avoidance

The CoM task is now written for the multi-robot CoM $c_{\text {multi-robot }}$, that is, the combined CoM of the system containing all the floating-base entities (robots, objects) in contact with each other. In the case of the robot manipulating a free-floating box, $c_{\text {multi-robot }}$ is the CoM of the $\{$ robot, box system. See Section III-C for more details on the CoM task and balancing during manipulation.

For the sake of simplicity, we showed here a particular instantiation of the general MRQP formulation: the simplified case in which the multi-robot system is restricted to one robot and one object. The general formulation in [16] with an arbitrary number $N$ of entities is much more expressive and allows control of multi-robot multi-object co-manipulation systems, where for example two robots collaborate to manipulate an object. We have implemented our approach within this general $N$-entity framework.

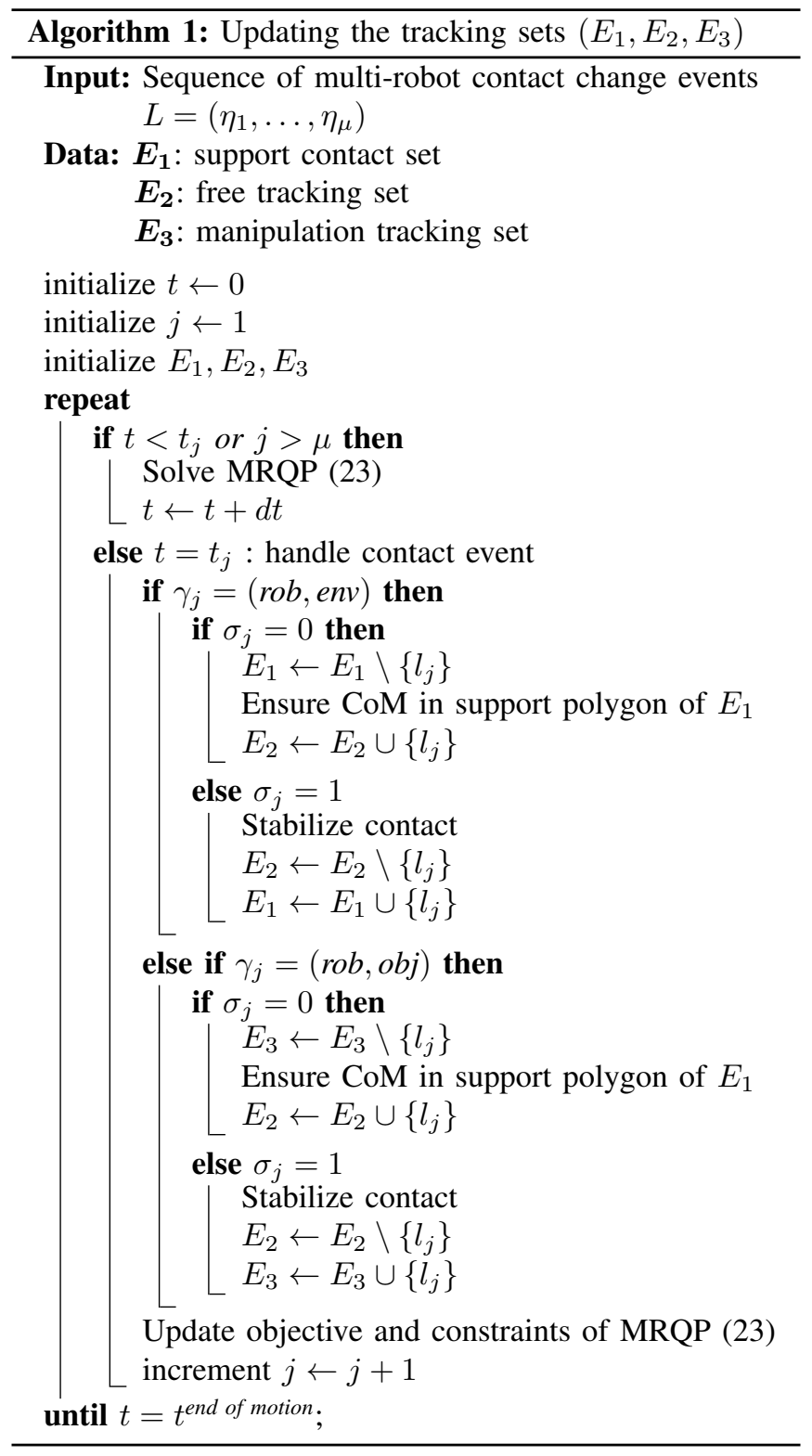

\section{B. Dealing with contact change events}

We adapt the motion capture tracking algorithm described at the end of Section II in the following way. The contact events $\eta_{j}$ now also encode the pair of entities between which the contact event happens, in a variable $\gamma_{j} \cdot \gamma_{j}$ takes a value in the set

$$
\gamma_{j} \in\{(\text { rob, env }),(\text { rob }, \text { obj }),(\text { obj, env })\} .
$$

$l_{j}$ now encodes the index of the link/surface of the first entity in $\gamma_{j}, s_{j}$ the index of the link/surface of the second entity in $\gamma_{j}$. Therefore, $\eta_{j}=\left(\gamma_{j}, l_{j}, s_{j}, t_{j}, \sigma_{j}\right)$. When time $t_{j}$ of a contact event is reached, all three sets $E_{1}, E_{2}$ and $E_{3}$ are updated and the multi-robot QP is reformulated. Algorithm 1 presents the overall logic of our tracking method.

When a contact event is detected, the robot temporarily pauses human motion tracking to ensure that the contact is handled before proceeding. 
For contact removals, this amounts to moving the CoM of the system into the new support polygon before removing the support. For example, when the demonstration motion goes from three contacts (e.g. two feet and one hand on the table) to two contacts (e.g. one hand and one foot), the robot must bring its CoM to be between the two planned support surfaces before removing the contact. Similarly, if the robot is about to release a manipulated object, it must first check that its individual (not multi-robot) CoM lies within its support polygon. For contact additions, we add two temporary end effector tasks to ensure a stable contact, as described in [7]: a surface vector stabilization task to ensure that the robot's end-effector surface is parallel to the object surface, and a surface transform task to drive the distance between the two surfaces to zero. Once the contact change conditions are fulfilled, the robot resumes tracking the demonstration.

In our experiments described below, we first record the human demonstration and play it back afterwards for the robot to track. However, our algorithm is compatible with real-time tracking, as long as there is a mechanism for contact event detection. One caveat is that (as mentioned in [15]), contact and CoM stabilization add delay to the tracking, so movements with contact changes occurring in rapid succession may be difficult to track. However, we can compensate for the delay by "buffering" the human demonstration trajectory.

\section{Center of Mass tracking}

In the previous work [7], the CoM task horizontal reference value was set independently of the human CoM at $h\left(E_{1}\right)$, the center of the support polygon formed by the ground projections of the contact surfaces in $E_{1}$ (we call those surfaces support surfaces). In Section III-A we presented a direct extension of this approach, adapted to manipulation retargeting and multi-robot $\mathrm{QP}$ formulation, by replacing the CoM of the robot with the CoM of the combined system \{robot, object\} when the robot is manipulating a free-floating object, $c_{\text {multi-robot }}$. In this section, we propose an additional approach that allows tracking of the human CoM motion during multi-contact (but nonmanipulation) phases, resulting in smoother and more robust contact support changes.

We formulate a generalization of the method proposed in Koenemann et al [15], where the robot tracks a normalized offset (denoted as $\phi$ ) between its support surfaces. We define a support point $p_{i}$ as the center of a support surface that is in contact with the fixed environment.

Let us denote $c_{x, y}^{\text {hum }}$ as the 2D ground projection of the human CoM. First, $c_{x, y}^{\text {hum }}$ is projected onto the vector from an arbitrary reference support $p_{0}^{\text {hum }}$ to each of the other support points $p_{i}^{\text {hum }}, i=1, \ldots, n_{\text {supports }}$, following Koenemann's formulation, to get the normalized offsets:

$$
\phi_{i}^{\text {hum }}=\frac{\left(c_{x, y}^{\text {hum }}-p_{0}^{\text {hum }}\right) \cdot\left(p_{i}^{\text {hum }}-p_{0}^{\text {hum }}\right)}{\left\|p_{i}^{\text {hum }}-p_{0}^{\text {hum }}\right\|^{2}} .
$$

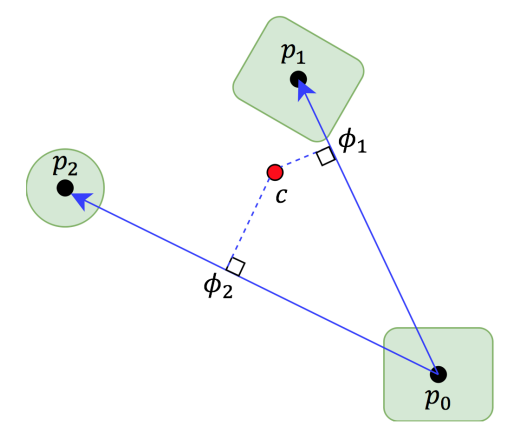

Fig. 2. Finding normalized offset values for CoM tracking. The contact surfaces correspond to a phase in the bag retrieval task in Section IV, with foot contacts $p_{0}$ and $p_{1}$, hand contact $p_{2}$.

The target coordinates for the robot's CoM projection can then be calculated as a linear combination of these offsets with:

$$
\psi\left(E_{1}, c_{x, y}^{\text {hum }}\right)=p_{0}^{\mathrm{rob}}+\sum_{i=0}^{n_{\text {supports }}} \phi_{i}^{\mathrm{hum}}\left(p_{i}^{\mathrm{rob}}-p_{0}^{\mathrm{rob}}\right) .
$$

We finally replace the reference CoM in (9) with

$$
c_{\text {multi-robot }, x, y}^{\text {ref }}=\psi\left(E_{1}, c_{x, y}^{\text {hum }}\right) .
$$

The process is illustrated in Fig. 2. In the case of double support, this amounts to the formulation of [15]. Our formulation generalizes to situations in which there are hands in contact with the environment. Compared to tracking CoM coordinates in Cartesian space, tracking the CoM in normalized coordinates is more robust to differences in robot size, as well as slight offsets in support position.

\section{EXPERIMENTS}

To demonstrate the advantages of our method, we conducted three whole-body manipulation tasks in simulation on the HRP-2 and HRP-4 humanoid robots. Two of the tasks require balancing while manipulating objects, and the other demonstrates a scenario with a non-free-floating environment object. Footage of both the human demonstrations and robot motions are in the supplementary video.

For each experiment, we first record a human demonstration to guide the robot in the task. We use an Xsens inertial motion capture suit to capture human pose. The demonstration data from the motion capture system is then annotated with contact change events (additions or removals of contacts points). In the experiments described below, this post-processing step is done manually, as it is out of the scope of this paper. However, there are many ways to extract contact event information as an offline step (as shown in [7]), or in real-time, using Xsens's contact detection API or force sensors on the human's end-effectors. After recording the human motion, we set up an identical environment for the humanoid robot in simulation, and have it use the algorithm described above to follow the human's demonstration.

In the first experiment, we demonstrate a motion to pick up a bag from a table while using the table as support to 

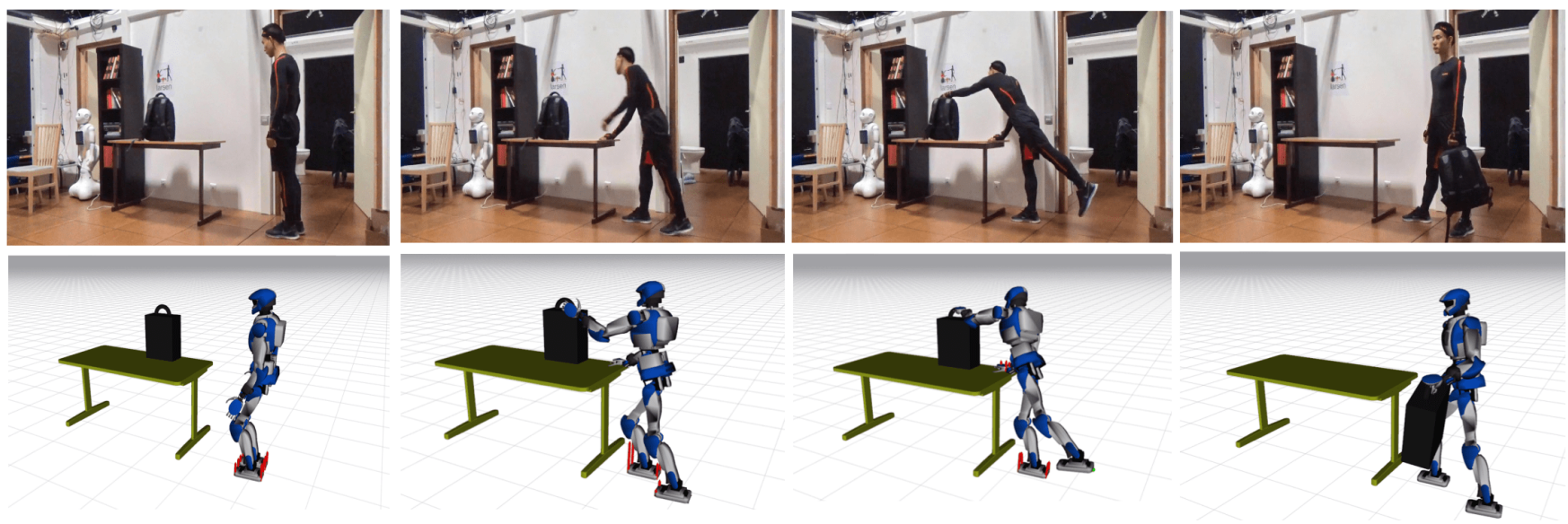

Fig. 3. First demonstration task: robot must use its hand as a support on the table while reaching for a bag.
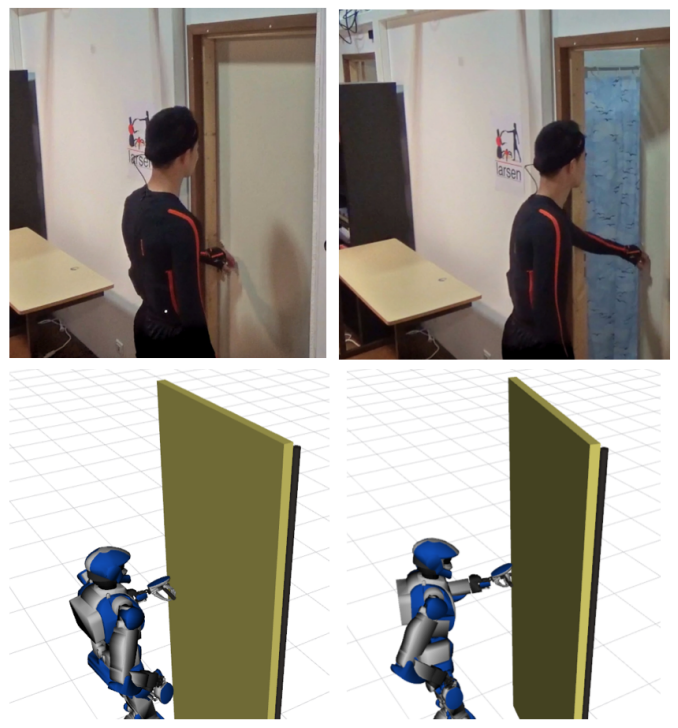

Fig. 4. Second task: opening a door.

lean forward (shown in Fig. 3). This task requires multiple interactions with both static and floating-base environment objects. For this particular task, we slowed down playback of the human demonstration motion to account for contact change events that violated our quasi-static CoM stability assumption. This will be discussed further in Sections V and VI.

For the second task, we retarget a door-opening motion to demonstrate a simple example of a non-floating-base environment object in the multi-robot QP. The human demonstration is carried out on a door whose hinge joint is not springloaded (spring constant $k=0$ ). In the simulation, we vary the spring constant of the door joint between $k=0,10,100$. Figure 5 shows the changes in actuation that the multi-robot QP computes in order to execute a visually similar motion on doors of varying resistance.

The third task demonstrates adaptation to a free-floating environment object of varying inertial parameters. It is se-

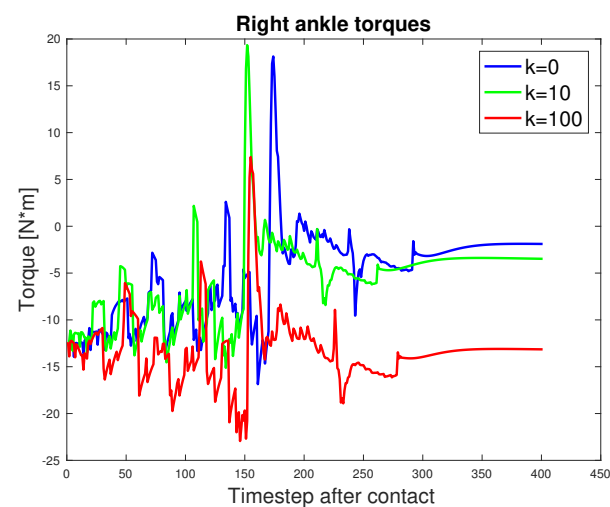

Fig. 5. Torques from right ankle during door opening task, for varying spring constants. Higher ankle torques for higher spring constants because the robot must "push" against the door's resistance.

quenced as follows: squat down, pick up a box from the floor, shift to balance on one leg, place box on table. This is depicted in Fig. 6. The human naturally tends to push the floating leg backwards to maintain their joint CoM within the support polygon and reach further with their arms.

The original demonstration was conducted with a box of mass $0.35 \mathrm{~kg}$. For the experiment, we varied the mass of the box to show the effects on the robot's behavior. The results are shown in Fig. 7. Note that as the box gets heavier, the robot moves its right foot further backwards to compensate and keep the combined CoM inside its singlefoot support polygon. In doing so, it "ignores" other lowerweight objectives such as human motion tracking (right foot position) and reference posture tracking. This behavior emerges naturally from the weighted prioritization scheme in the QP. For this task, using a single-robot QP (without the box incorporated into the dynamics model) fails, because the robot does not compensate for the gravitational force acting on the box.

In Fig. 8, we plot the motor torque commands output by the multi-robot QP (23). It is clear that as the box gets heavier, the controller compensates with higher torques in 

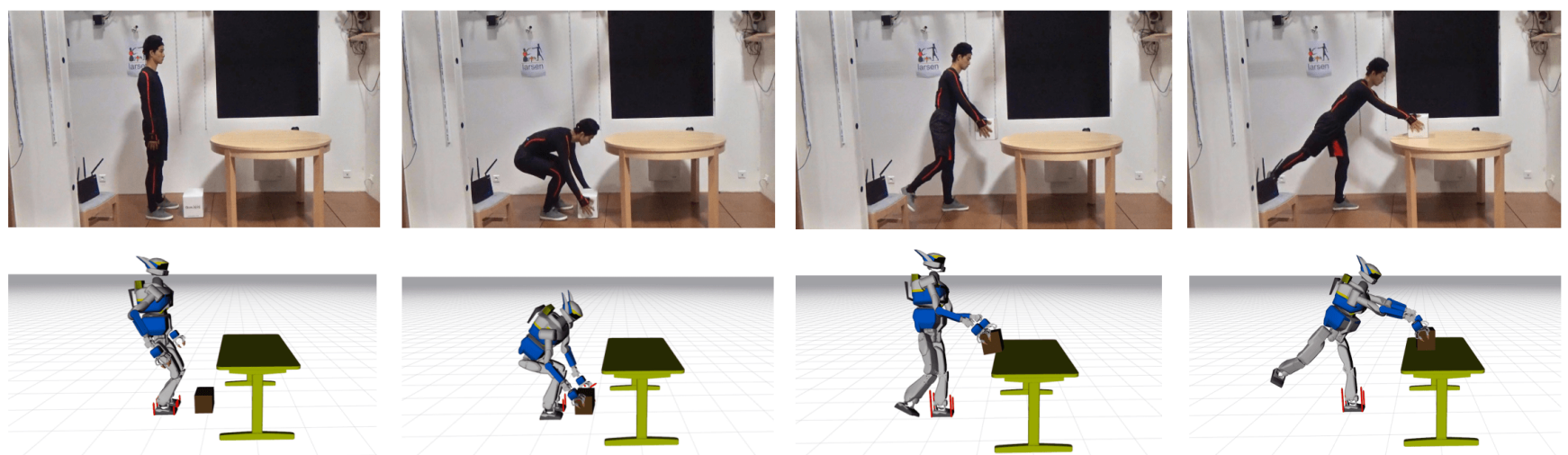

Fig. 6. Third demonstration task: picking up a box, balancing on one foot and placing on table

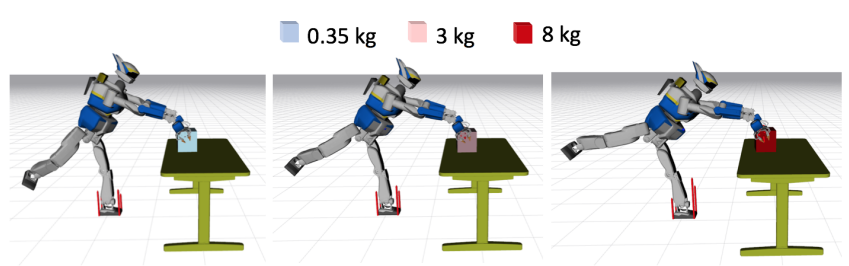

Fig. 7. The robot following a single human demonstration for boxes of varying mass: $0.35 \mathrm{~kg}$ (left), $3 \mathrm{~kg}$ (center), $8 \mathrm{~kg}$ (right). Notice that the robot moves its right foot backward to compensate for the heavier box, maintaining the combined CoM position.
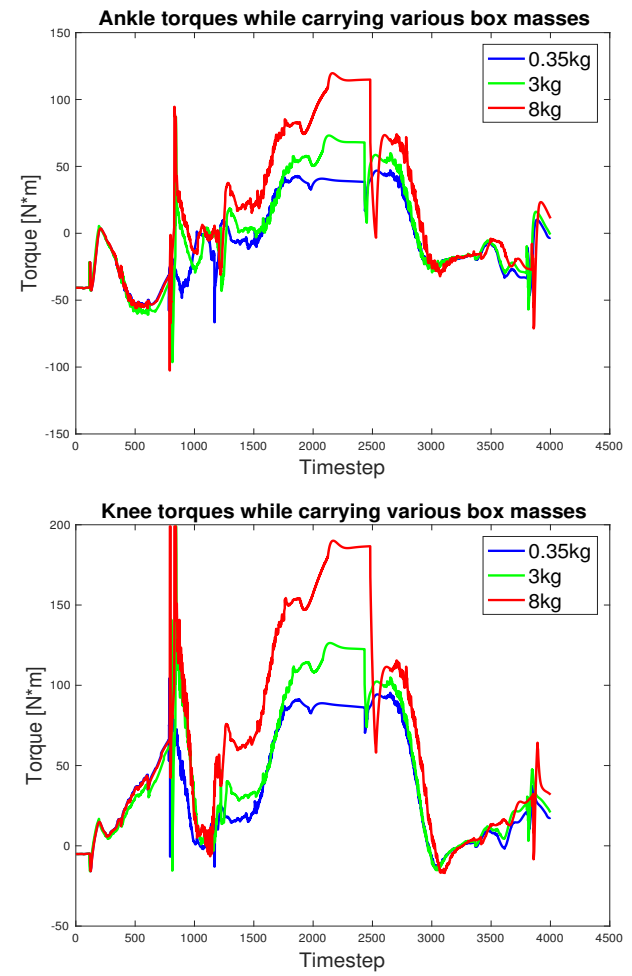

Fig. 8. Motor torques in left leg during the third experiment. A clear difference can be seen in the motor torque commands for each box weight the lower body, even with visually similar motions. Although our robots use position-controlled joints (a setpoint $q_{\text {des }}$ set by double-integrating $\ddot{q}$ output from QP), this would be useful as feed-forward torque commands for torque-controlled robots.

\section{CONCLUSions}

In this work, we have presented a framework using a multi-robot quadratic program for adaptive whole-body manipulation tasks in human-to-humanoid motion retargeting. The incorporation of the dynamics of all "robots" into a single optimization allows for automatic adaptation of the human demonstration to environment objects of varying physical properties. This ability to retarget motions involving heavy object manipulation is novel, and a contribution to the state-of-the-art. Our controller also opens up possibilities for collaborative motions between robot-robot and human-robot teams. We demonstrated the capabilities of our framework through simulation experiments.

An unsolved issue is the tracking of highly dynamic motions with contact set changes. Such dynamic motions are usually achieved in humanoids with offline computationally trajectory optimization approaches [20] based on non-linear optimization tools [21]. In our experiments, we assume that the human's demonstrations can be tracked with quasistatically stable motions - hence our conditions for bringing the CoM above the support polygon before removing contacts. Another limitations is that the system does not yet run in real-time (addressed below in Future Work). We also make assumptions about the robot operating in a known environment with a model provided by the human operator (this was the case, for example, in the DARPA Robotics Challenge). However, there has been progress towards automatic extraction of environment model parameters using a Kalman-filter approach [17], friction coefficients from vision [22], and specially designed neural networks [23].

\section{FUTURE WORK}

In the near future, we will implement the algorithm presented here on a real humanoid robot. We plan on using force controllers on the end effectors (as described in [17]) to account for differences between expected and real-world 
contact forces. We will also integrate touch-sensing gloves and shoes so that contact events can be detected on-the-fly and used for real-time motion tracking.

Many common human motions are not quasistatically stable; in fact, they use momentum to achieve better energy efficiency. In humanoid robots, this insight has led to planning and execution of dynamic locomotion using simplified models such as ZMP [24] and centroidal momentum [25]. However, these methods often assume full authority over the robot's future movements. The difficulty with tracking human motions in real-time is that the human dictates the reference motion for the robot, and the robot does not know where they will go in the future. We believe that the first step towards highly dynamic motion tracking would be to adopt some of the methods based on simplified models used in locomotion planning, such as the capture point [26]. Examining the contact wrench set is also a good way to measure feasibility, and there has been some recent work in constructing a feasible region of CoM positions and accelerations based on this notion [27]. In the longer term, we envision the combination of a fully versatile human-tohumanoid loco-manipulation retargeting system with wholebody motor-imagery BCI control [28] to enhance the retargeting system with a feed-forward predictive "intention of motion" component.

\section{ACKNOWLEDGEMENTS}

The authors would like to thank the IDH team in LIRMM and JRL for their support in using the multi-robot QP framework.

\section{REFERENCES}

[1] N. S. Pollard, J. K. Hodgins, M. J. Riley, and C. G. Atkeson, "Adapting human motion for the control of a humanoid robot," in Robotics and Automation, 2002. Proceedings. ICRA'02. IEEE International Conference on, vol. 2. IEEE, 2002, pp. 1390-1397.

[2] B. Dariush, M. Gienger, A. Arumbakkam, Y. Zhu, B. Jian, K. Fujimura, and C. Goerick, "Online transfer of human motion to humanoids," International Journal of Humanoid Robotics, vol. 6, no. 02, pp. 265-289, 2009.

[3] K. Yamane and J. Hodgins, "Control-aware mapping of human motion data with stepping for humanoid robots," in Intelligent Robots and Systems (IROS), 2010 IEEE/RSJ International Conference on. IEEE, 2010, pp. 726-733.

[4] S.-K. Kim, S. Hong, and D. Kim, "A walking motion imitation framework of a humanoid robot by human walking recognition from imu motion data," in Humanoid Robots, 2009. Humanoids 2009. 9th IEEE-RAS International Conference on. IEEE, 2009, pp. 343-348.

[5] K. Hu, C. Ott, and D. Lee, "Online human walking imitation in task and joint space based on quadratic programming," in Robotics and Automation (ICRA), 2014 IEEE International Conference on. IEEE, 2014, pp. 3458-3464.

[6] G. Bin Hammam, P. M. Wensing, B. Dariush, and D. E. Orin, "Kinodynamically consistent motion retargeting for humanoids," International Journal of Humanoid Robotics, vol. 12, no. 04, p. 1550017, 2015.

[7] A. Di Fava, K. Bouyarmane, K. Chappellet, E. Ruffaldi, and A. Kheddar, "Multi-contact motion retargeting from human to humanoid robot," in Humanoid Robots (Humanoids), 2016 IEEE-RAS 16th International Conference on. IEEE, 2016, pp. 1081-1086.

[8] K. Bouyarmane and A. Kheddar, "On weight-prioritized multitask control of humanoid robots," IEEE Transactions on Automatic Control, 2017.

[9] Y. Abe, M. Da Silva, and J. Popović, "Multiobjective control with frictional contacts," in Proceedings of the 2007 ACM SIGGRAPH/Eurographics symposium on Computer animation. Eurographics Association, 2007, pp. 249-258.
[10] M. De Lasa, I. Mordatch, and A. Hertzmann, "Feature-based locomotion controllers," in ACM Transactions on Graphics (TOG), vol. 29, no. 4. ACM, 2010, p. 131.

[11] K. Bouyarmane, J. Vaillant, F. Keith, and A. Kheddar, "Exploring humanoid robots locomotion capabilities in virtual disaster response scenarios," in Humanoid Robots (Humanoids), 2012 12th IEEE-RAS International Conference on. IEEE, 2012, pp. 337-342.

[12] S. Feng, X. Xinjilefu, C. G. Atkeson, and J. Kim, "Optimization based controller design and implementation for the atlas robot in the darpa robotics challenge finals," in Humanoid Robots (Humanoids), 2015 IEEE-RAS 15th International Conference on. IEEE, 2015, pp. 10281035.

[13] S. Kuindersma, R. Deits, M. Fallon, A. Valenzuela, H. Dai, F. Permenter, T. Koolen, P. Marion, and R. Tedrake, "Optimization-based locomotion planning, estimation, and control design for the atlas humanoid robot," Autonomous Robots, vol. 40, no. 3, pp. 429-455, 2016.

[14] Y. Abe and J. Popović, "Interactive animation of dynamic manipulation," in Proceedings of the 2006 ACM SIGGRAPH/Eurographics symposium on Computer animation. Eurographics Association, 2006, pp. $195-204$.

[15] J. Koenemann, F. Burget, and M. Bennewitz, "Real-time imitation of human whole-body motions by humanoids," in Robotics and Automation (ICRA), 2014 IEEE International Conference on. IEEE, 2014, pp. 2806-2812.

[16] J. Vaillant, K. Bouyarmane, and A. Kheddar, "Multi-character physical and behavioral interactions controller," IEEE transactions on visualization and computer graphics, vol. 23, no. 6, pp. 1650-1662, 2017.

[17] K. Bouyarmane, J. Vaillant, K. Chappellet, and A. Kheddar, "Multirobot and task-space force control with quadratic programming," Mar. 2017, working paper or preprint. [Online]. Available: https://hal.archives-ouvertes.fr/hal-01495662

[18] E. S. Ho, T. Komura, and C.-L. Tai, "Spatial relationship preserving character motion adaptation," ACM Transactions on Graphics (TOG), vol. 29 , no. 4 , p. $33,2010$.

[19] S. Nakaoka and T. Komura, "Interaction mesh based motion adaptation for biped humanoid robots," in Humanoid Robots (Humanoids), 2012 12th IEEE-RAS International Conference on. IEEE, 2012, pp. 625631.

[20] S. Lengagne, J. Vaillant, E. Yoshida, and A. Kheddar, "Generation of whole-body optimal dynamic multi-contact motions," The International Journal of Robotics Research, vol. 32, no. 9-10, pp. 1104-1119, 2013.

[21] T. Moulard, F. Lamiraux, K. Bouyarmane, and E. Yoshida, "Roboptim: an optimization framework for robotics," in Robomec, 2013, p. 4p.

[22] M. Brandao, K. Hashimoto, and A. Takanishi, "Friction from vision: A study of algorithmic and human performance with consequences for robot perception and teleoperation," in Humanoid Robots (Humanoids), 2016 IEEE-RAS 16th International Conference on. IEEE, 2016, pp. 428-435.

[23] G. Martius and C. H. Lampert, "Extrapolation and learning equations," arXiv preprint arXiv:1610.02995, 2016.

[24] S. Kajita, F. Kanehiro, K. Kaneko, K. Fujiwara, K. Harada, K. Yokoi, and $\mathrm{H}$. Hirukawa, "Biped walking pattern generation by using preview control of zero-moment point," in Robotics and Automation, 2003. Proceedings. ICRA'03. IEEE International Conference on, vol. 2. IEEE, 2003, pp. 1620-1626.

[25] H. Dai, A. Valenzuela, and R. Tedrake, "Whole-body motion planning with centroidal dynamics and full kinematics," in Humanoid Robots (Humanoids), 2014 14th IEEE-RAS International Conference on. IEEE, 2014, pp. 295-302.

[26] J. Pratt, J. Carff, S. Drakunov, and A. Goswami, "Capture point: A step toward humanoid push recovery," in Humanoid Robots, 2006 6th IEEE-RAS International Conference on. IEEE, 2006, pp. 200-207.

[27] H. Audren and A. Kheddar, "3D robust stability polyhedron in multi-contact," Feb. 2017. [Online]. Available: https://hallirmm.ccsd.cnrs.fr/lirmm-01477362

[28] K. Bouyarmane, J. Vaillant, N. Sugimoto, F. Keith, J.-i. Furukawa, and J. Morimoto, "Brain-machine interfacing control of whole-body humanoid motion," Frontiers in systems neuroscience, vol. 8, 2014. 